\title{
Correction to: The first case of Planococcus glaciei found in blood, a report from the Czech Republic
}

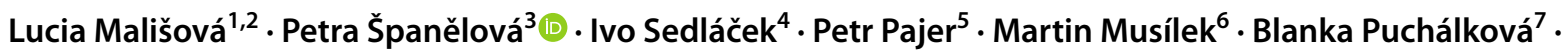 \\ Vladislav Jakubů ${ }^{1,2,8} \cdot$ Helena Žemličková ${ }^{1,2,8} \cdot$ Renáta Šafránková ${ }^{3}$
}

Published online: 9 December 2021

(c) Institute of Microbiology, Academy of Sciences of the Czech Republic, v.v.i. 2021

\section{Correction to: Folia Microbiologica (2021) https://doi.org/10.1007/s12223-021-00919-4}

Incomplete and thus hard-to-understand versions of Tables 1 and 2 were published in the original Online First article. Footnotes, unfortunately, had appeared at the end of the text part.

This erratum provides well organized, correct and easyto-understand version of the two above-mentioned tables.

The Authors apologize to readers for any inconvenience.

The original article can be found online at https://doi.org/10.1007/ s12223-021-00919-4.

Petra Španělová

petra.spanelova@szu.cz

1 National Reference Laboratory for Antibiotics, National Institute of Public Health, Prague, Czech Republic

2 Department of Microbiology, 3Rd Faculty of Medicine, Charles University, University Hospital Kralovske Vinohrady and National Institute of Public Health, Prague, Czech Republic

3 Czech National Collection of Type Cultures, National Institute of Public Health, Prague, Czech Republic

4 Department of Experimental Biology, Faculty of Science, Czech Collection of Microorganisms, Masaryk University, Brno, Czech Republic
5 Military Health Institute, Military Medical Agency, Prague, Czech Republic

6 National Reference Laboratory for Meningococcal Infections, National Institute of Public Health, Prague, Czech Republic

7 Department of Clinical Microbiology, Karlovy Vary Regional Hospital Czech Republic, Prague, Czech Republic

8 Department of Clinical Microbiology, Faculty of Medicine and University Hospital, Charles University, Hradec Kralove, Czech Republic 


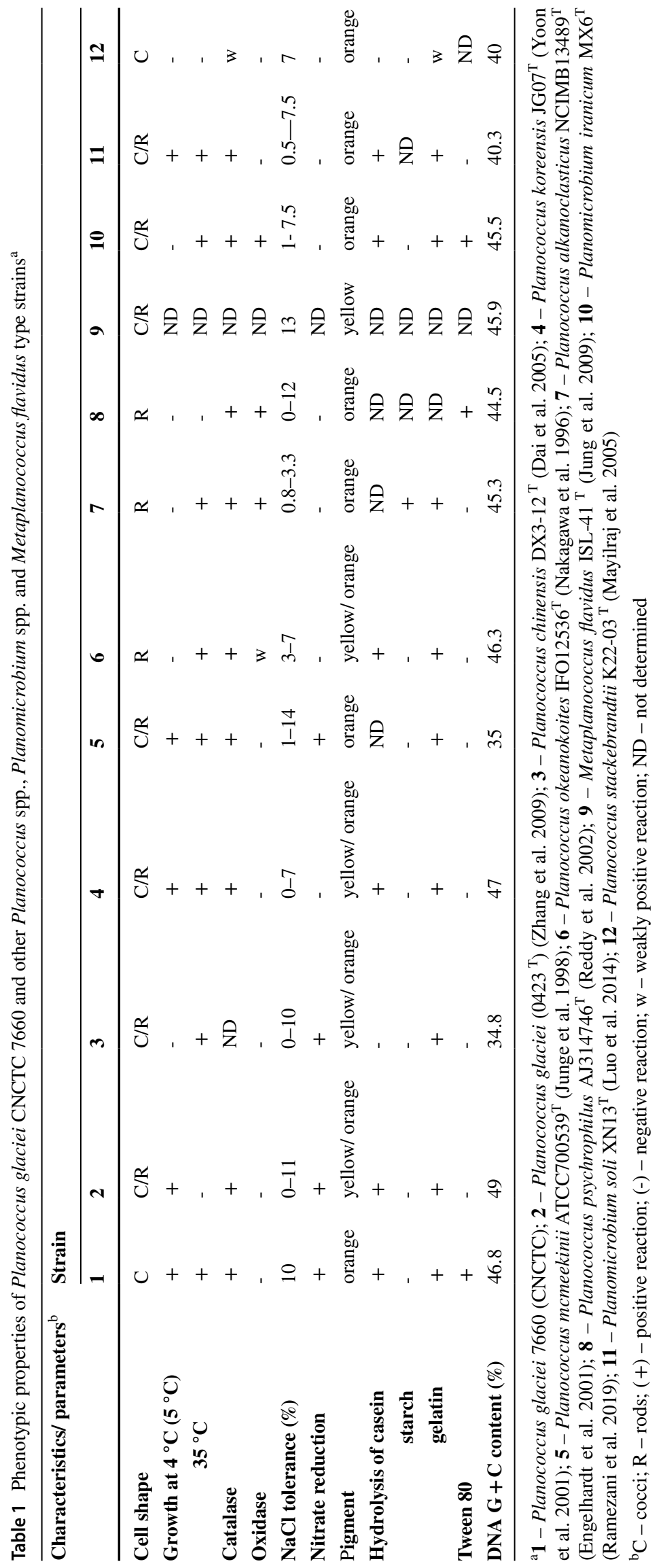


Table 2 Biochemical characteristics of Planococcus glaciei, strains CNCTC 7660 and $0423^{\mathrm{T}}$ (data acquired from Zhang et al. 2009), results of API ${ }^{2} Z Y M$ and $A P I \circledast 20 N E$

\begin{tabular}{|c|c|c|}
\hline \multirow[t]{2}{*}{ Characteristics/parameters $^{\mathrm{a}}$} & \multicolumn{2}{|l|}{ Strain } \\
\hline & $\begin{array}{l}\text { P. glaciei } \\
7660\end{array}$ & $\begin{array}{l}P . \\
\text { glaciei } \\
\mathbf{0 4 2 3}^{\mathrm{T}}\end{array}$ \\
\hline \multicolumn{3}{|l|}{ API@ZYM } \\
\hline alkaline phosphatase & - & + \\
\hline esterase $(\mathbf{C 4})$ & - & ND \\
\hline esterase lipase $(\mathrm{C} 8)$ & - & + \\
\hline lipase & - & ND \\
\hline leucine arylamidase & + & + \\
\hline valine arylamidase & + & + \\
\hline cystine arylamidase & - & + \\
\hline trypsin & - & + \\
\hline$\alpha$-chymotrypsin & + & + \\
\hline acid phosphatase & - & - \\
\hline naphthol-AS-BI-phosphohydrolase & - & + \\
\hline$\alpha$-galactosidase & - & - \\
\hline$\beta$-galactosidase & - & + \\
\hline$\beta$-glucuronidase & - & - \\
\hline$\alpha$-glucosidase & - & - \\
\hline$\beta$-glucosidase & - & - \\
\hline $\mathrm{N}$-acetyl- $\beta$-glucosaminidase & - & ND \\
\hline$\alpha$-mannosidase & - & ND \\
\hline$\alpha$-fucosidase & - & - \\
\hline \multicolumn{3}{|l|}{ API®20NE } \\
\hline D-mannose & - & + \\
\hline glucose & - & + \\
\hline L-arabinose & - & + \\
\hline mannitol & - & + \\
\hline N-acetyl-glucosamine & - & ND \\
\hline maltose & - & ND \\
\hline gluconate & - & ND \\
\hline caprate & - & ND \\
\hline adipate & - & ND \\
\hline malate & - & ND \\
\hline citrate & - & ND \\
\hline phenyl-acetate & - & ND \\
\hline
\end{tabular}

ND - not determined

${ }^{\mathrm{a}}(+)$ - positive reaction; $(-)$ - negative reaction

Publisher's Note Springer Nature remains neutral with regard to jurisdictional claims in published maps and institutional affiliations. 